Pacific Journal of Mathematics

THE CONNECTED COMPONENT OF THE IDÈLE CLASS
GROUP OF AN ALGEBRAIC NUMBER FIELD 


\title{
THE CONNECTED COMPONENT OF THE IDELE CLASS GROUP OF AN ALGEBRAIC NUMBER FIELD
}

\section{MIDORI KOBAYASHI}

\begin{abstract}
We shall give another proof of Weil's theorem of the structure of the connected component of the idèle class group of an algebraic number field. Our proof is different from Artin's.
\end{abstract}

Let $Q$ be the rational number field and $k$ be an algebraic number field of finite degree over $Q$. We denote by $C_{k}$ the idèle class group of $k$ and $D_{k}$ the connected component of unity of $C_{k}$. Let $T$ denote the multiplicative group of all complex numbers of absolute value 1 with compact topology, $R$ the additive group of the real numbers with usual topology, and $S$ the Solenoid with compact topology.

Weil ([3]) has shown that $D_{k}$ is isomorphic to $T^{r_{2}} \times R \times S^{r}$, by determining the structure of the dual $D_{k}^{*}$. Artin ([1]) has exhibited a system of representatives of idèle classes and given a different proof. In this paper we shall give another proof of the above Weil's theorem.

1. Let $k$ be an algebraic number field which has $r_{1}$ real infinite primes and $r_{2}$ complex infinite primes. As usual we put $r=r_{1}+r_{2}-1$. Let $I_{k}$ be the idèle group of $k, C_{k}$ the idèle class group of $k$ and $D_{k}$ the connected component of unity of $C_{k}$. An idèle will be denoted by $\left(a_{v}\right)=\left(a_{\mathfrak{p}}, a_{\lambda}\right)$, where $v$ runs all primes of $k, \mathfrak{p}$ all finite primes and $\lambda$ all infinite primes of $k\left(\lambda=1, \ldots, r_{1}+r_{2}\right)$. We shall agree that $\lambda\left(1 \leq \lambda \leq r_{1}\right)$ is real and $\lambda\left(r_{1}+1 \leq \lambda \leq r_{1}+r_{2}\right)$ is complex. Let us denote by $\sigma_{\lambda}$ the embedding of $k$ into the complex number field attached to an infinite prime $\lambda$. Then $\sigma_{\lambda}$ with $1 \leq \lambda \leq r_{1}$ is a real embedding and $\sigma_{\lambda}$ with $r_{1}+1 \leq \lambda \leq r_{1}+r_{2}$ a complex one.

For any topological group $G, G^{*}$ denotes the character group of $G$. If $\chi$ is a character of $C_{k}$, i.e., a continuous homomorphism of $C_{k}$ into $T$, we can regard it as a character of $I_{k}$ which is trivial on principal idèles. If we restrict $\chi$ to the infinite part $R^{\times r_{1}} C^{\times^{r_{2}}}$ of $I_{k}, \chi$ can be written as follows:

$$
\chi\left(\left(a_{\lambda}\right)\right)=\prod_{\lambda=1}^{r_{1}+r_{2}}\left(\frac{a_{\lambda}}{\left|a_{\lambda}\right|}\right)^{f_{\lambda}}\left|a_{\lambda}\right|^{\sqrt{-1} \varphi_{\lambda}}, \quad\left(a_{\lambda}\right) \in R^{\times^{r_{1}}} C^{\times^{r_{2}}},
$$

where $f_{\lambda} \in Z$ (the rational integers), $\varphi_{\lambda} \in R\left(\lambda=1, \ldots, r_{1}+r_{2}\right)$, and $f_{1}, \ldots, f_{r_{1}}=0$ or 1 . Such $f_{\lambda}$ and $\varphi_{\lambda}\left(\lambda=1, \ldots, r_{1}+r_{2}\right)$ are uniquely determined, so we say that $\chi$ is of type $\left(f_{\lambda}, \varphi_{\lambda}\right)$. 
It is well known that the following two lemmas hold.

LEMma 1. Let $\chi$ be a character of $C_{k}$, of type $\left(f_{\lambda}, \varphi_{\lambda}\right)$. Then the conditions (i), (ii) and (iii) are equivalent to each other:

(i) $\chi$ is of finite order.

(ii) $\chi\left(D_{k}\right)=1$.

(iii) $f_{\iota}=0\left(\iota=r_{1}+1, \ldots, r_{1}+r_{2}\right), \varphi_{\lambda}=0\left(\lambda=1, \ldots, r_{1}+r_{2}\right)$.

Lemma 2. Let $f_{\lambda} \in Z$ and $\varphi_{\lambda} \in R\left(\lambda=1, \ldots, r_{1}+r_{2}\right)$, where $f_{1}, \ldots, f_{r_{1}}$ $=0$ or 1 . Then the statements (i) and (ii) are equivalent to each other:

(i) There exists a character of $C_{k}$, of type $\left(f_{\lambda}, \varphi_{\lambda}\right)$.

(ii) Define the character $\mathrm{X}$ of the unit group $E$ of $k$ as follows, then $\mathrm{X}$ is of finite order:

$$
\mathrm{X}(\varepsilon)=\prod_{\lambda=1}^{r_{1}+r_{2}}\left(\frac{\varepsilon^{\sigma_{\lambda}}}{\left|\varepsilon^{\sigma_{\lambda}}\right|}\right)^{f_{\lambda}}\left|\varepsilon^{\sigma_{\lambda}}\right|^{\sqrt{-1} \varphi_{\lambda}}, \quad \varepsilon \in E .
$$

2. In order to show that the isomorphism $D_{k}^{*}=Z^{r_{2}} \times R \times Q^{r}$ as topological groups, consider a homomorphism $\mathcal{H}$ of $C_{k}^{*}$ to the additive group $Z^{r_{2}} \times R^{r_{1}+r_{2}}$. For any $\chi \in C_{k}^{*}$, if $\chi$ is of type $\left(f_{\lambda}, \varphi_{\lambda}\right)$, we put $\mathscr{H}(\chi)=\left(f_{\iota}, \varphi_{\lambda}\right)$ : the element of $Z^{r_{2}} \times R^{r_{1}+r_{2}}$, where $\iota=r_{1}+1, \ldots, r_{1}+r_{2}$ and $\lambda=1, \ldots, r_{1}+r_{2}$; as $f_{1}, \ldots, f_{r_{1}}=0$ or 1 , they are neglected. It is clear that $\mathcal{H}$ is a homomorphism (algebraically) whose kernel is $T_{k}$ from Lemma 1. We denote by $M$ the image of $\mathcal{H}$ in $Z^{r_{2}} \times R^{r_{1}+r_{2}}$.

3. Let $\varepsilon_{1}, \ldots, \varepsilon_{r}$ be a system of fundamental units of $k$. Let $f_{\iota} \in Z$ $\left(\iota=r_{1}+1, \ldots, r_{1}+r_{2}\right)$ and $\varphi_{\lambda} \in R\left(\lambda=1, \ldots, r_{1}+r_{2}\right)$. Assume that $\left(f_{\iota}, \varphi_{\lambda}\right)$ belongs to $M$. From Lemma 2 , the character $\mathrm{X}$ of $E$,

$$
\mathrm{X}(\varepsilon)=\prod_{\iota=r_{1}+1}^{r_{1}+r_{2}}\left(\frac{\varepsilon^{\sigma_{\iota}}}{\left|\varepsilon^{\sigma_{\imath}}\right|}\right)^{f_{\iota} r_{1}+r_{2}} \prod_{\lambda=1}^{\varepsilon^{\sigma_{\lambda}}} \mid \varepsilon^{\sqrt{-1}} \varphi_{\lambda}, \quad \varepsilon \in E,
$$

is of finite order; for, if $\lambda$ is a real infinite prime, $\varepsilon^{\sigma_{\lambda}} /\left|\varepsilon^{\sigma_{\lambda}}\right|$ is always \pm 1 , so $f_{\lambda}\left(\lambda=1, \ldots, r_{1}\right)$ can be neglected. Then there exists an integer $n$ such that

$$
\mathrm{X}\left(\varepsilon_{1}^{n}\right)=\mathrm{X}\left(\varepsilon_{2}^{n}\right)=\cdots=\mathrm{X}\left(\varepsilon_{r}^{n}\right)=1,
$$

which means

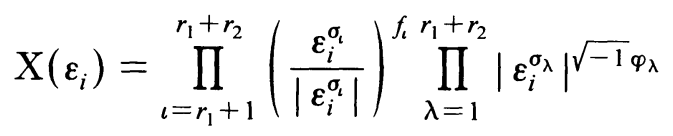


is a root of unity $(i=1, \ldots, r)$. Put $\varepsilon_{i}^{\sigma_{\iota}} /\left|\varepsilon_{i}^{\sigma_{t}}\right|=e^{\sqrt{-1} \theta_{u}}\left(\iota=r_{1}+1, \ldots, r_{2}\right.$; $i=1, \ldots, r)$, then

$$
\begin{aligned}
X\left(\varepsilon_{i}\right) & =\prod_{\iota=r_{1}+1}^{r_{1}+r_{2}} e^{\sqrt{-1} \theta_{u} f_{\iota}} \prod_{\lambda=1}^{r_{1}+r_{2}} e^{\sqrt{-1} \varphi_{\lambda} \log \mid \varepsilon_{\imath}^{\sigma_{\imath} \lambda \mid}} \\
& =e^{\sqrt{-1}\left(\Sigma_{\imath} \theta_{u} f_{\iota}+\Sigma_{\lambda} \varphi_{\lambda} \log \left|\varepsilon_{\iota}{ }^{\sigma_{\lambda}}\right|\right)}
\end{aligned}
$$

so we have

$$
\sum_{\iota=r_{1}+1}^{r_{1}+r_{2}} \theta_{i \iota} f_{\iota}+\sum_{\lambda=1}^{r_{1}+r_{2}} \varphi_{\lambda} \log \left|\varepsilon_{i}^{\sigma_{\lambda}}\right| \in \pi Q, \quad(i=1, \ldots, r) .
$$

Further we put $\beta_{i \iota}=\theta_{\iota \iota} / \pi$ and $\alpha_{i \lambda}=\left(\log \left|\varepsilon_{i}^{\sigma_{\lambda}}\right|\right) / \pi\left(i=1, \ldots, r ; \iota=r_{1}+\right.$ $\left.1, \ldots, r_{1}+r_{2} ; \lambda=1, \ldots, r_{1}+r_{2}\right)$, then we obtain

$$
\sum_{\iota=r_{1}+1}^{r_{1}+r_{2}} \beta_{i \iota} f_{\iota}+\sum_{\lambda=1}^{r_{1}+r_{2}} \alpha_{i \lambda} \varphi_{\lambda} \in Q \quad(i=1, \ldots, r) .
$$

The converse is immediate. Hence, for any $\left(f_{\iota}, \varphi_{\lambda}\right) \in Z^{r_{2}} \times R^{r_{1}+r_{2}},\left(f_{\iota}, \varphi_{\lambda}\right)$ belongs to the group $M$ if and only if $\left(f_{\iota}, \varphi_{\lambda}\right)$ satisfies the condition (\#).

As $\left\{\varepsilon_{1}, \ldots, \varepsilon_{r}\right\}$ is a system of fundamental units, we should notice that the following hold:

(i) $\alpha_{i 1}+\cdots+\alpha_{i r_{1}}+2 \alpha_{i r_{1}+1}+\cdots+2 \alpha_{i r_{1}+r_{2}}=0(i=1, \ldots, r)$,

(ii) $\operatorname{det}\left(\alpha_{i j}\right)_{1 \leq i, j \leq r} \neq 0$.

Now we shall show that $M$ is isomorphic to the additive group $Z^{r_{2}} \times R \times Q^{r}$ as abstract groups. There uniquely exists $\left(x_{1}, \ldots, x_{r}\right) \in R^{r}$ satisfying $\beta_{i r_{1}+1}+\alpha_{i 1} x_{1}+\cdots+\alpha_{i r} x_{r}=0(i=1, \ldots, r)$ from (ii). Put

$$
N_{1}=\left\{(\underbrace{f, 0, \ldots, 0}_{r_{2}} ; \underbrace{f x_{1}, \ldots, f x_{r}, 0}_{r_{1}+r_{2}}) \mid f \in Z\right\}
$$

and

$$
\begin{array}{r}
M_{1}=\left\{(\underbrace{0, f_{r_{1}+2}, \ldots, f_{r_{1}+r_{2}}}_{r_{2}} ; \underbrace{\varphi_{1}, \ldots, \varphi_{r_{1}+r_{2}}}_{r_{1}+r_{2}}) \in Z^{r_{2}} \times R^{r_{1}+r_{2}},\right. \\
\quad \operatorname{satisfying}(\#)\},
\end{array}
$$

then $N_{1}$ and $M_{1}$ are subgroups of $M$ and $N_{1}$ is isomorphic to $Z$. We have immediately a direct decomposition $M=N_{1} \times M_{1} \cong Z \times M_{1}$, for any element of $M\left(f_{r_{1}+1}, \ldots, f_{r_{1}+r_{2}} ; \varphi_{1}, \ldots, \varphi_{r_{1}+r_{2}}\right)$ is written as $\left(f_{r_{1}+1}\right.$, 
$\left.0, \ldots, 0 ; f_{r_{1}+1} x_{1}, \ldots, f_{r} x_{r}, 0\right)+\left(0, f_{r_{1}+2}, \ldots, f_{r_{1}+r_{2}} ; \varphi_{1}-f_{r_{1}+1} x_{1}, \ldots, \varphi_{r}-\right.$ $\left.f_{r} x_{r}, \varphi_{r_{1}+r_{2}}\right) \in N_{1} \times M_{1}$. Let $\left(y_{1}, \ldots, y_{r}\right)$ be the element of $R^{r}$ satisfying $\beta_{i r_{1}+2}+\alpha_{i 1} y_{1}+\cdots+\alpha_{i r} y_{r}=0(i=1, \ldots, r)$. Put

$$
\begin{gathered}
N_{2}=\left\{(\underbrace{0, f, 0, \ldots, 0}_{r_{2}} ; \underbrace{f y_{1}, \ldots, f y_{r}, 0}_{r_{1}+r_{2}}) \mid f \in Z\right\}, \\
M_{2}=\left\{(\underbrace{0,0, f_{r_{1}+3}, \ldots, f_{r_{1}+r_{2}}}_{r_{2}} ; \underbrace{\varphi_{1}, \ldots, \varphi_{r_{1}+r_{2}}}_{r_{1}+r_{2}}) \in Z^{r_{2}} \times R^{r_{1}+r_{2}},\right. \\
\text { satisfying (\#) }\},
\end{gathered}
$$

then we have immediately that $M_{1}=N_{2} \times M_{2} \cong Z \times M_{2}$ in the same way, which implies $M \cong Z^{2} \times M_{2}$. By induction we obtain that $M \cong Z^{r_{2}}$ $\times M_{r_{2}}$, where

$$
\begin{array}{r}
M_{r_{2}}=\left\{(\underbrace{0, \ldots, 0}_{r_{2}} ; \varphi_{1}, \ldots, \varphi_{r_{1}+r_{2}}) \mid \alpha_{i 1} \varphi_{1}+\cdots+\alpha_{i r_{1}+r_{2}} \varphi_{r_{1}+r_{2}} \in Q\right. \\
(i=1, \ldots, r)\} .
\end{array}
$$

Put

$$
N^{\prime}=\left\{(\underbrace{0, \ldots, 0}_{r_{2}} ; \underbrace{\varphi / 2, \ldots, \varphi / 2}_{r_{1}}, \underbrace{\varphi, \ldots, \varphi}_{r_{2}}) \mid \varphi \in R\right\},
$$

then, from (i), $N^{\prime}$ is a subgroup of $M_{r_{2}}$; isomorphic to $R$. And put $N^{\prime \prime}=\left\{(\underbrace{0, \ldots, 0}_{r_{2}} ; \underbrace{\varphi_{1}, \ldots, \varphi_{r}, 0}_{r_{1}+r_{2}}) \mid \alpha_{i 1} \varphi_{1}+\cdots+\alpha_{i r} \varphi_{r} \in Q(i=1, \ldots, r)\right\}$,

then $N^{\prime \prime}$ is a subgroup of $M_{r_{2}}$, and it is clear that the map $(0, \ldots, 0$; $\left.\varphi_{1}, \ldots, \varphi_{r}, 0\right) \rightarrow\left(b_{1}, \ldots, b_{r}\right)$, satisfying

$$
\left(\begin{array}{ccc}
\alpha_{11} & \cdots & \alpha_{1 r} \\
\vdots & & \vdots \\
\alpha_{r 1} & \cdots & \alpha_{r r}
\end{array}\right)\left(\begin{array}{c}
\varphi_{1} \\
\vdots \\
\varphi_{r}
\end{array}\right)=\left(\begin{array}{c}
b_{1} \\
\vdots \\
b_{r}
\end{array}\right) \text {, }
$$

is an isomorphism of $N^{\prime \prime}$ onto $Q^{r}$ from (ii): $N^{\prime \prime} \cong Q^{r}$. It immediately follows that

$$
M_{r_{2}}=N^{\prime} \times N^{\prime \prime} \cong R \times Q^{r} .
$$


Thus we obtain the isomorphism as abstract groups

$$
M \cong Z^{r_{2}} \times R \times Q^{r} \text {. }
$$

4. Identifying $M$ with $Z^{r_{2}} \times R \times Q^{r}$ through the algebraic isomorphism, the map $\mathcal{H}: C_{k}^{*} \rightarrow M$, defined in 2, is as follows:

$$
\begin{aligned}
C_{k}^{*} & \rightarrow Z^{r_{2}} \times R \times Q^{r} \\
\chi\left(\text { of type }\left(f_{\lambda}, \varphi_{\lambda}\right)\right) & \rightarrow(\underbrace{*, \ldots, *}_{r_{2}} ; \varphi_{r_{1}+r_{2}}, \underbrace{*, \ldots, *}_{r}) .
\end{aligned}
$$

We shall agree that $R$ has the usual topology and that $Z$ and $Q$ have discrete topology. If we show the map $\mathcal{H}$ is open and continuous, we have the isomorphism as topological groups $C_{k}^{*} / T_{k}=Z^{r_{2}} \times R \times Q^{r}$ because the kernel of $\mathcal{H}$ is $T_{k}$ as mentioned in 2. By Lemma $1 C_{k}^{*} / T_{k} \cong D_{k}^{*}$, which shows $D_{k}^{*} \cong Z^{r_{2}} \times R \times Q^{r}$, and hence we have from the duality theorem

$$
D_{k} \cong T^{r_{2}} \times R \times S^{r} \text {. }
$$

We shall show that the map $\mathcal{H}$ is open and continuous. Let $C_{k}^{0}$ be the set of all idèle classes with volume 1 , and $A$ the set of all idèle classes of $\left(a_{v}\right)$ which has component $a_{\lambda_{r_{1}+r_{2}}}$ at the infinite prime $\lambda_{r_{1}+r_{2}}$ and all other components equal to 1 . As is well-known it holds that $C_{k} \stackrel{\lambda_{1}+r_{2}}{=} A \times C_{k}^{0}$. We denote by $A^{*}$ and $\left(C_{k}^{0}\right)^{*}$ the group of all characters of $C_{k}$ which is trivial on $C_{k}^{0}$ and $A$, respectively, and then we have $C_{k}^{*}=A^{*} \times\left(C_{k}^{0}\right)^{*}$.

Now for any real number $\varphi$ and any ideal $a$ of $k$, we define

$$
\psi(\mathfrak{a})=\operatorname{Norm}(\mathfrak{a})^{-\sqrt{-1} \varphi / 2},
$$

then $\psi$ is a Grössencharakter mod 1. For any principal ideal $(\gamma), \gamma \in k^{\times}$, we can see

$$
\psi((\gamma))=\prod_{\lambda=1}^{r_{1}}\left|\gamma^{\sigma_{\lambda}}\right|^{-\sqrt{-1} \varphi / 2} \prod_{\lambda=r_{1}+1}^{r_{1}+r_{2}}\left|\gamma^{\sigma_{\lambda}}\right|^{-\sqrt{-1} \varphi},
$$

so $\psi$ is of type

$$
(\underbrace{0, \ldots, 0}_{r_{2}} ; \underbrace{-\varphi / 2, \ldots,-\varphi / 2}_{r_{1}}, \underbrace{-\varphi, \ldots,-\varphi}_{r_{2}}) .
$$

We denote by $\chi_{\psi}$ the character of $C_{k}$ associated with $\psi$, i.e., for any idèle $a=\left(a_{v}\right)$

$$
\chi_{\psi}(a)=\psi(\operatorname{id}(a)) \prod_{\lambda=1}^{r_{1}}\left|a_{\lambda}\right|^{\sqrt{-1} \varphi / 2} \prod_{\lambda=r_{1}+1}^{r_{1}+r_{2}}\left|a_{\lambda}\right|^{\sqrt{-1} \varphi},
$$


it is of type

$$
(\underbrace{0, \ldots, 0}_{r_{2}} ; \underbrace{\varphi / 2, \ldots, \varphi / 2}_{r_{1}}, \underbrace{\varphi, \ldots, \varphi}_{r_{2}}) .
$$

Each idèle $a=\left(a_{v}\right)$ determines in an obvious manner an ideal of $k$, and so denote it by $\operatorname{id}(a)$.

Let $Y$ be the set of all such characters $\chi_{\psi}: Y=\left\{\chi_{\psi} \mid \varphi \in R\right\}$. As is well-known, a character $\chi_{\psi}$ defined as mentioned above is trivial on $C_{k}^{0}$, and the converse is also valid, that is $Y=A^{*}$. For any character $\chi_{\psi} \in Y$ of type $(0, \ldots, 0 ; \varphi / 2, \ldots, \varphi / 2, \varphi, \ldots, \varphi)$ and any idèle $a=\left(a_{v}\right) \in A$, we can see

$$
\chi_{\psi}(a)= \begin{cases}a_{\lambda_{r_{1}+r_{2}}}^{\sqrt{-1} \varphi / 2} & \left(\text { if } \lambda_{r_{1}+r_{2}} \text { is a real prime, i.e., } r_{2}=0\right), \\ a_{\lambda_{r_{1}+r_{2}}}^{\sqrt{-1} \varphi} & \left(\text { if } \lambda_{r_{1}+r_{2}} \text { is a complex prime, i.e., } r_{2} \neq 0\right) .\end{cases}
$$

$A$ with the relative topology of $C_{k}$ is isomorphic to $R_{+}^{\times}$(the multiplicative group of positive real numbers). Therefore the map $\chi_{\psi} \rightarrow \varphi$ is an isomorphism of $A^{*}=Y$ with the relative topology of $C_{k}^{*}$ to the additive group $R$ as topological groups: $Y \cong R$.

We restrict the map $\mathcal{H}$ on $Y$, then we have (cf. $(*))$

$$
\begin{gathered}
Y \rightarrow Z^{r_{2}} \times R \times Q^{r} \\
\chi_{\psi} \rightarrow(\mathbf{0}, \varphi, \mathbf{0}) .
\end{gathered}
$$

It is open and continuous because $\chi_{\psi} \rightarrow \varphi$ is a topological isomorphism as mentioned above. Since $\left(C_{k}^{0}\right)^{*}, Z$ and $Q$ are discrete, the map

$$
\mathcal{H}: C_{k}^{*}=Y \times\left(C_{k}^{0}\right)^{*} \rightarrow Z^{r_{2}} \times R \times Q^{r}
$$

is clearly open and continuous. This completes the proof.

\section{REFERENCES}

1. E. Artin, Representatives of the connected component of idele class group, Proceedings of International Symposium on Algebraic Number Theory at Tokyo-Nikko (1955), 51-54.

2. A. Weil, On a certain type of characters of the idele-class group of an algebraic number field, ibid., 1-7.

3. A. Weil, Sur la thèorie du corps de classes, J. Math. Soc. Japan, 3 (1951), 1-35.

Received October 1, 1981.

NAGASAKI UNIVERSITY

NAGASAKI, JAPAN 


\section{PACIFIC JOURNAL OF MATHEMATICS EDITORS}

DONALD BABBITT (Managing Editor)

University of California

Los Angeles, CA 90024

Hugo Rossi

University of Utah

Salt Lake City, UT 84112

C. C. Moore and Arthur Ogus

University of California

Berkeley, CA 94720
J. DugunduI

Department of Mathematics

University of Southern California

Los Angeles, CA 90089-1113

R. FinN and H. SAmelson

Stanford University

Stanford, CA 94305

\section{ASSOCIATE EDITORS}
R. ARENS
E. F. BECKENBACH
B. H. NeUmaNN
F. WolF
K. YoshidA (1906-1982)

\section{SUPPORTING INSTITUTIONS}

UNIVERSITY OF ARIZONA

UNIVERSITY OF BRITISH COLUMBIA

CALIFORNIA INSTITUTE OF TECHNOLOGY

UNIVERSITY OF CALIFORNIA

MONTANA STATE UNIVERSITY

UNIVERSITY OF NEVADA, RENO

NEW MEXICO STATE UNIVERSITY

OREGON STATE UNIVERSITY
UNIVERSITY OF OREGON

UNIVERSITY OF SOUTHERN CALIFORNIA

STANFORD UNIVERSITY

UNIVERSITY OF HAWAII

UNIVERSITY OF TOKYO

UNIVERSITY OF UTAH

WASHINGTON STATE UNIVERSITY

UNIVERSITY OF WASHINGTON 


\section{Pacific Journal of Mathematics}

\section{Vol. 106, No. $1 \quad$ November, 1983}

John Ballard, Clifford's theorem for algebraic groups and Lie algebras . . . . . 1

Philip Richard Bartick, II and Edwin Duda, Finite-to-one open mappings

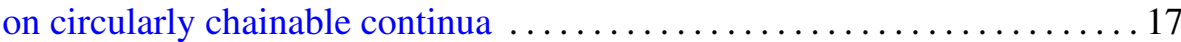

Frank Hayne Beatrous, Jr., $H^{\infty}$-interpolation from a subset of the

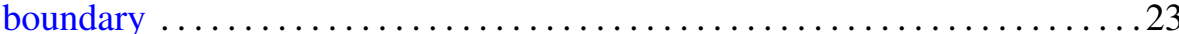

Sterling K. Berberian, Expectations in semifinite algebras ............ 33

Heron S. Collins and Wolfgang Ruess, Weak compactness in spaces of compact operators and of vector-valued functions $\ldots \ldots \ldots \ldots \ldots \ldots . \ldots 45$

David Downing and William O. Ray, Renorming and the theory of

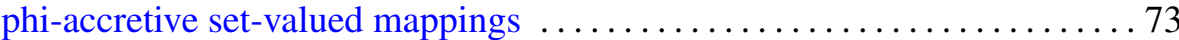

Harvey Charles Greenwald, On the theory of homogeneous Lipschitz

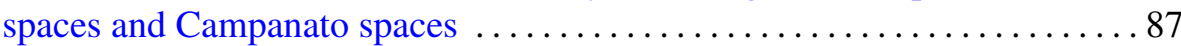

Irwen Valle Guadalupe and Lucio Ladislao Rodriguez, Normal curvature

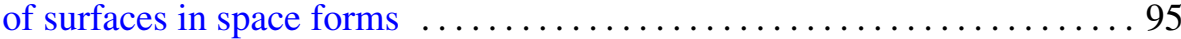

Boju Jiang, On the computations of the Nielsen number ............... 105

William H. Julian, Ray Mines, III and Fred Richman, Alexander

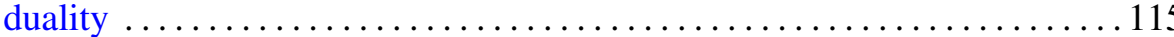

Midori Kobayashi, The connected component of the idèle class group of an

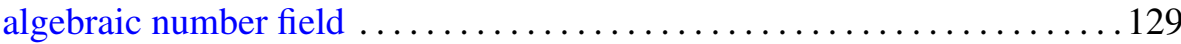

Ib Henning Madsen, Charles B. Thomas and C. Terence C. (Charles)

(Clegg) Wall, Topological spherical space form problem. III.

Dimensional bounds and smoothing

A. Sapounakis, The existence of strong liftings for totally ordered measure

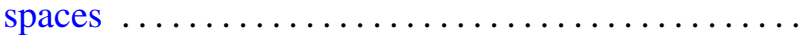

Gary Seitz, The root subgroups for maximal tori in finite groups of Lie type

Laurent Siebenmann and James M. Van Buskirk, Construction of irreducible homology 3-spheres with orientation reversing involution 